\title{
El síndrome del recomendado
}

\author{
A. SANZ RUBIALES, $M^{\mathrm{a}}$. L. DEL VALLE RIVERO, L. A. FLORES PÉREZ*, \\ S. HERNANSANZ DE LA CALLE, C. GARCÍA RECIO, F. LÓPEZ-LARA MARTÍN
}

\author{
Hospital Universitario de Valladolid. *Centro de Salud de Medina del Campo. Valladolid
}

\author{
SYNDROME OF RECOMMENDED PATIENT
}

\begin{abstract}
RESUMEN
El "síndrome del recomendado" se manifiesta como la aparición de imprevistos y de complicaciones no habituales en pacientes con los que se pretende desarrollar una atención más esmerada. Aparte de lo que pueda ser debido al azar, hay factores de la práctica clínica habitual que favorecen la manifestación de este síndrome, muchos de los cuales se pueden corregir. Todos provienen del cambio de la práctica habitual que se da con estos pacientes, como si en ellos no fuera adecuado seguir la misma actitud que con los demás. Algunos de los factores que favorecen la aparición de este síndrome son: la actitud del propio paciente, el empleo ineficiente de los recursos sanitarios, la ausencia de un registro adecuado de datos en la historia clínica y el cambio en la conducta habitual en la indicación y la interpretación de los estudios diagnósticos y en el tratamiento de estos enfermos. El mejor modo de prevenir este "síndrome del recomendado" es mantener, también con estos pacientes, una actitud clínica basada en unos conocimientos sólidos y seguir la misma línea de conducta que con los demás enfermos.
\end{abstract}

PALABRAS CLAVE: Errores médicos. Yatrogenia. Sensibilidad. Especificidad.

\begin{abstract}
"Syndrome of recommended patient" is manifested as the presence of numerous unexpected and unusual complications in patients that the treating physician is trying to give a better assistance. Even assuming that a few complications may appear by chance, there are several factors from daily clinical practice that facilitate the presence of such a syndrome, and some of them can be corrected in order to reduce its incidence. All of them come from the change on daily clinical practice on these patients, as if they do not fit for the attention provided for other people. These factors favouring the presence of this syndrome come from: patients' attitude, inefficient use of health resources, absence of an ade quate register of clinical data and change in usual clinical practice on interpretation of diagnostic tests as well as in the indication of treatment of these patients. The best way to prevent this "syndrome of recommen ded patient" is to maintain, even within these patients, an attitude based on solid clinical knowledge and to follow up the same clinical rules accepted for other patients.
\end{abstract}

KEY WORDS: Medical errors. Iatrogenic disease. Sensitivity. Specificity. recomendado. An Med Interna (Madrid) 2002; 19: 430-433.

\section{INTRODUCCIÓN}

El concepto médico de "síndrome", es decir, "conjunto de síntomas característicos de una enfermedad", se aplica a diversos avatares del quehacer clínico diario que no son propiamente entidades nosológicas pero que se suelen presentar de manera similar. Uno de los que se pueden encontrar en la práctica habitual es lo que se ha dado en llamar "síndrome del recomendado". En este término se condensa la experiencia común que enseña que las personas a las que se pretende facilitar una atención no ya mejor, de acuerdo con el principio de justicia, sino especial, más personalizada o con mayor esmero padecen, de manera "incomprensible", complicaciones y sucesos inesperados. Sin embargo, algo que sucede de manera reiterada en condiciones similares no se debería achacar siempre al azar. Por tanto, es preciso analizar nuestra actuación ante estos pacientes para conocer los posibles errores que se puedan estar cometiendo. Y para intentar corregirlos.

En este trabajo intentamos enumerar los signos y "síntomas" habituales de este peculiar "síndrome del recomendado". A continuación se proponen algunas de los factores que favorecen su aparición. Y, por último, se ofrecen unas estrategias de actuación dirigidas a prevenirlo. Ninguna de las enumeraciones se puede considerar exhaustiva. Al contrario, se trata más bien de un estímulo para animar a que cada uno aporte otras manifestaciones, otras causas y otras vías para prevenir el "síndrome del recomendado".

Trabajo aceptado: 15 de abril de 2002

Correspondencia: Álvaro Sanz Rubiales. Hospital Universitario de Valladolid. C/ Ramón y Cajal 3. 47011 Valladolid. e-mail: asrubiales@ hotmail.com 


\section{MANIFESTACIONES}

En resumen, el "síndrome del recomendado" se podría definir como la aparición de complicaciones no habituales y de imprevistos, muchos ellos difíciles de explicar, en pacientes concretos en los que la intención es llevar a cabo una atención más esmerada y personalizada ya que son personas de mayor renombre o han sido recomendados por motivos de posición social, fama, parentesco, etc.

Este síndrome se puede manifestar de diversas maneras. A continuación se presenta una serie, no exhaustiva, de los signos y de las manifestaciones principales que le son característicos. En todo caso, para poder definirlo no es preciso que aparezcan todos ellos. Estamos convencidos de que cada profesional podrá añadir a las que se presentan otras expresiones de este síndrome que habrá podido observar en su propia experiencia.

- La evolución de la enfermedad, del proceso diagnóstico o del problema que se trate suele ser más tórpida y con más complicaciones que las que cabría esperar en un paciente en condiciones similares.

-El paciente sufre una yatrogenia, derivada tanto de tratamientos médicos como quirúrgicos, superior a la que se presenta habitualmente en otros pacientes en las mismas circunstancias.

-Aparecen retrasos y olvidos en las citaciones para consultas y estudios complementarios. En más de una ocasión estos estudios se llevan a cabo en condiciones inadecuadas.

-Los registros clínicos de la evolución del paciente y los estudios complementarios que se le hayan podido realizar se extravían y/o son difíciles de localizar. Cuando se encuentran, suelen aportar una información demasiado escueta.

\section{PATOGENIA}

El "síndrome del recomendado" es la consecuencia de muchas buenas intenciones que, de manera involuntaria, se acompañan de una actuación poco acertada (1). Por desgracia, que los buenos deseos no siempre vengan acompañados de buenos resultados se debe a que los medios que se emplean tal vez no sean los más adecuados (2).

Se proponen algunas causas que pueden favorecer que se manifieste este síndrome (Tabla I). Todas ellas provienen de un origen común: ante estos pacientes se pierde la costumbre y la mecánica, la "rutina buena", de la práctica habitual, como si con ellos no fuera adecuado seguir los mismos pasos sencillos y minuciosos que en cualquier otro paciente. En general, de los demás factores, no hay ninguno que sea imprescindible para que se presente. Aunque es posible que se desarrolle sin que se dé ninguna de ellas, es realmente raro que el "síndrome del recomendado" aparezca por una mera cuestión de "mala suerte".

\section{LOS PACIENTES}

—Rechazan verse incluido en la "rutina" de la práctica habitual.

- Suelen mostrar una excesiva confianza en las aptitudes del médico al que se recurre (3) y en los efectos beneficiosos del tratamiento (4). Se prefiere confiar la atención a las personas de mayor prestigio y mayor antigüedad aunque este presti-
TABLA I

PATO GENIA DEL “SÍNDRO ME DEL RECO M ENDADO ”

O rigen de las causas del "síndrome del recomendado"

Solicitud de una atención especial por parte de los pacientes

Desorden en el empleo de los recursos sanitarios

M ala calidad en el registro de datos; extravío o fragmentación de la historia clínica

Ausencia de líneas comunes de actuación y de liderazgo en el personal sanitario

Exceso de estudios diagnósticos con los consiguientes "falsos positivos" O misión de estudios sencillos habituales

Sobretratamiento y yatrogenia

gio puede no ser un reflejo de su experiencia reciente. A la vez, se desconfía de los profesionales más jóvenes.

-El paciente no conoce toda la información sobre su enfermedad, especialmente si el pronóstico es malo o incierto. Sin embargo, esta información se suele facilitar a la persona que recomienda o "avala" al paciente, que a su vez opina sobre la conveniencia de informar o no al paciente (5).

-Las propias características del paciente, con el interés y el esmero que provoca, hacen que los sucesos que en otros enfermos se asumirían como "normales" (retrasos, olvidos, extravíos, problemas de comunicación) en este caso se vean agrandados, como con un "efecto lupa", y se sientan como más severos y más relevantes.

\section{LA UTILIZACIÓN DE LOS RECURSOS}

- Se atiende a los pacientes fuera del lugar físico y del horario habitual (en los pasillos, en las guardias etc.), sin disponibilidad directa de los recursos habituales del Hospital. Y sin posibilidad de dejar registrados los hallazgos de la exploración o de los estudios complementarios o las decisiones que se adoptan.

- Las citaciones para consultas y estudios complementarios, se realizan de palabra y sin registro documental ("sin pasar por la ventanilla"). Con lo que quedan a expensas de la memoria y de la buena voluntad de las personas que se comprometen verbalmente a llevarlas a cabo, a veces sin tener en cuenta otros compromisos.

- Las citaciones para consultas y estudios complementarios, en más de una ocasión se retrasan, se olvidan o se llevan a cabo en malas condiciones. Es decir, fuera de los lugares y horarios habituales, con la consecuente pérdida de calidad e información, en contra de las previsiones.

- Cambio parcial o total en los horarios y en las prácticas habituales de cuidado del enfermo para respetar su intimidad o evitar la sensación de enfermedad y hospitalización. De esta forma se sale de la rutina habitual, lo que puede llevar a omitir pasos importantes para la orientación diagnóstica y el tratamiento.

- La presencia de un paciente notable, fuera de hora y sin citación, puede traducirse en una mayor presión sobre el personal sanitario y en un retraso y una peor atención de pacien- 
tes citados. Lo que origina quejas y un incremento aun mayor del stress.

- Si precisa ingreso hospitalario, se lleva a cabo en una planta o en una sala de mayor comodidad pero no especializada en la patología específica del paciente.

\section{EL REGISTRO DE DATOS EN LA HISTORIA CLÍNICA}

-No se elabora una historia clínica adecuada. Se omiten las informaciones que provienen de estudios poco elaborados. Raramente se dispone de informes que reseñen la evolución y, sobre todo, la situación actual del paciente.

- La historia clínica no aparece cuando se busca en el archivo correspondiente. Si aparece, no suele contener un registro adecuado de la situación clínica, la evolución y la actitud terapéutica. Los registros de la historia clínica se fragmentan entre los diferentes profesionales que atienden a un paciente.

- Los estudios complementarios se informan de manera urgente, con frecuencia de manera oral, sin un registro adecuado. Cuando hay un registro documental no siempre se incorpora a la historia clínica sino que queda directamente de la mano del médico, del paciente o de los familiares.

\section{EL PERSONAL SANITARIO}

-Falta una dirección explícita en la conducta clínica. No se identifica un responsable último de la actuación y de las decisiones. Es posible que lo hubiera al inicio del tratamiento pero que haya desistido al encontrarse tantos colegas opinando y matizando sus opiniones, más o menos acertadas. A esto se añade una mayor sensación de sentirse observado ("examinado") por parte del paciente y de otros colegas.

-Intervienen, opinan, colaboran y, en más de una ocasión, dirigen el tratamiento varios médicos de manera prácticamente simultánea. Unos por obligación, otros por motivo de relaciones familiares o de prestigio, algunos por solicitud del paciente o la familia y un buen número por el mero deseo de colaborar. Se desglosa la patología del paciente en cada una de sus vertientes de manera que especialistas diferentes asumen el control de cada uno de estos problemas. Con frecuencia no hay una comunicación adecuada, falta una persona de referencia y, con ello, una orientación común.

- Las líneas de actuación son variadas e incluso contradictorias porque se marcan al ritmo de la inspiración de los médicos, fuera de los protocolos habituales de tratamiento (6). Esto es algo que critican incluso los refranes que cristalizan la sabiduría popular ("un médico, cura; dos, dudan; tres, muerte segura") (7).

- La atención se deja en manos de facultativos de mayor prestigio y mayor antigüedad (8). Incluso cuando su experiencia clínica y su capacidad para el manejo de ciertas patologías pueda no ser la más adecuada. Es conocido que en algunos profesionales de renombre los conocimientos clínicos se centran en un área diferente de la especialidad o el prestigio se debe al tiempo dedicado a trabajos sin trato directo con el enfermo, como investigación (clínica y/o básica), docencia y formación.

- Se desconfía de los profesionales jóvenes. No se tiene en cuenta que pueden atender frecuentemente y con solvencia patologías más complejas.
-Adquieren mayor validez las "consultas de pasillo" en las que otro médico, sin necesidad de comprometerse, orienta la actitud ante un caso clínico que se le plantea. Sin embargo, estos comentarios pueden aportar una visión sesgada ya que quien los pronuncia desconoce al paciente y no asume responsabilidades directas en las decisiones clínicas.

-Otros médicos que deberían colaborar en el diagnóstico y/o en el tratamiento limitan su participación para evitar equivocarse y ser juzgados por otros profesionales.

-El médico con parentesco con el paciente pasa a asumir de manera inconsciente, tanto por su parte como por parte del resto del personal sanitario, el papel de médico de referencia. Aunque la patología no se encuentre dentro de su ámbito de capacitación, es a él a quien se explican las actitudes y de quien se solicita la toma de decisiones.

\section{LOS ESTUDIOS DIAGNÓSTICOS}

-Falta interés y falta información sobre los antecedentes personales y la exploración física, en ocasiones incluso sobre los datos más relevantes (alergias, medicación habitual, etc.).

-Los estudios complementarios solicitados pueden no ser los más indicados. Se pueden encontrar dos tendencias. Por una parte la de evitar estudios agresivos, aunque puedan ser convenientes, con la intención de no incomodar al paciente. Por otra parte, se puede tender a pruebas diagnósticas cruentas que originan una yatrogenia excesiva.

- Los estudios diagnósticos se repiten de manera innecesaria. Se estima que junto con las técnicas habituales, en estos pacientes es conveniente realizar otros más modernos o que se estiman más precisos. La reiteración de estudios facilita que se encuentren falsos positivos. Y que, de acuerdo con estos resultados, se instauren nuevos tratamientos (9).

- Los criterios habituales de sensibilidad y de especificidad de los estudios diagnósticos se alteran. El médico tiende a sospechar con más facilidad la presencia de determinadas patologías con lo que inconscientemente se aumenta la sensibilidad y, por tanto, el porcentaje de falsos positivos (10).

\section{EL TRATAMIENTO}

- Se emplean opciones terapéuticas múltiples, a veces sin una línea clara, con intención de cubrir todos los problemas del paciente (demostrados, sospechados y predecibles).

-El empleo de técnicas novedosas y sofisticadas se interpreta como una muestra de excelencia en la atención clínica. Sin embargo, en estas técnicas se tiene menos experiencia y pueden no haber demostrado aún un beneficio clínico relevante en relación con otras más contrastadas (11). Cuando estos tratamientos más novedosos y sofisticados producen yatrogenia, aparece un problema añadido ya que se hace más difícil atenderlos puesto que incluso los médicos más solventes carecen de experiencia en estas complicaciones.

- Llega un momento en que es muy difícil conocer qué problemas se deben a la enfermedad y cuáles están inducidos por los diferentes tratamientos. Además, es fácil entrar en una espiral en que estas complicaciones inducen a emplear todavía más fármacos (12).

- Se adopta una actitud activa ante los diferentes problemas médicos, con lo que se exacerba el "sesgo de tratamien- 
to" (13). En caso de duda se tiende a tratar antes que a "esperar y ver": es difícil retrasar u omitir tratamientos, aun los de eficacia dudosa (14). Además, la indicación de los tratamientos, médicos o quirúrgicos, se adapta al principio de "más es mejor" que defiende actitudes más agresivas (15).

\section{PREVENCIÓN}

En los "recomendados" se procura a dar la misma atención pero con más esmero. Esto no debería contraponerse con el imperativo de aplicar el criterio de justicia en el trato con todos los pacientes. De hecho, el mejor modo de prevenir el "síndrome del recomendado" es intentar mantener unas líneas de actuación básicas comunes a todos los enfermos. Y examinar periódicamente la propia conducta ante estos pacientes para detectar y corregir las desviaciones que se puedan introducir en la práctica habitual (16).

Hay unos pasos elementales que se deberían mantener también en el caso de estos pacientes y que ayudan a prevenir el "síndrome del recomendado":

- Realizar desde el primer día una historia clínica que reúna antecedentes personales, anamnesis por aparatos, historia del proceso actual y exploración física. En concreto no se debe obviar esta exploración por un falso pudor o por respeto. Este componente tradicional del acto médico no se consigue sustituir con los modernos estudios analíticos o de imagen.

-Emplear la historia clínica y archivar en ella una copia de todos los estudios complementarios y de los comentarios sobre la evolución clínica. Remitir esta historia al Archivo oportuno siempre que se haya empleado.

-Mantener un orden de horarios y citaciones. Guardar un registro ordenado de todos ellos donde comprobar las fechas y el cumplimiento del programa.

- Identificar en todo momento a quien hace cabeza, dirige o supervisa todo el tratamiento. Es decir, también los pacientes más relevantes deben tener un médico responsable, que sea el punto de referencia (para el paciente, para la familia y para el resto del personal sanitario) que unifique y que mantenga un criterio común.

\section{Bibliografía}

1. Hayward RA, Hofer TP. Estimating hospital deaths due to medical errors: preventability is in the eye of the reviewer. JAMA 2001; 286: 415-20.

2. Murillo E. A modo de guía para perplejos. Oncología 1992;15:156.

3. The AM, Hak T, Koëter G, van der Wal G. Collusion in doctor-patient communication about imminent death: an ethnographic study. BMJ 2000; 321: 1376-81.

4. Thornton H, Baum M. Ethics of clinical trials: the forbidden fruit phenomena. Breast 1996; 5: 1-4.

5. Fallowfield L. Participation of patients in decisions about treatment for cancer. BMJ 2001; 323: 1144.

6. Bonfill X, Gabriel R, Cabello J. La medicina basada en la evidencia. Rev Esp Cardiol 1997; 50: 819-25.

7. Martínez Kleiser L. Refranero General Ideológico Español. Madrid: Editorial Hernando, S.A.; 1993

8. Isaacs D, Fitzgerald D. Seven alternatives to evidence-based medicine. Oncologist 2001; 6: 390-1.
- Mantener los mismos criterios de prudencia de cara a los estudios diagnósticos y a los tratamientos que se emplean con los demás pacientes.

-Evitar la repetición inútil de estudios. Solicitar la opinión de especialistas no vinculados con el caso para que den una valoración no condicionada de los resultados de determinadas pruebas diagnósticas.

- Cuando el entorno del paciente empuja a desviarse a actitudes no contrastadas, es preciso mantener las líneas habituales de tratamiento con un respaldo suficiente tanto en la práctica común como bibliográfico, de "medicina basada en la evidencia" (17).

En resumen, la mejor prevención del "síndrome del recomendado" es el estudio, que permite tener conocimientos clínicos sólidos, e intentar mantener las líneas de conducta habituales, la "sana rutina", que ayuda a sacar adelante la labor clínica de cada día.

\section{CONCLUSIÓN}

El "síndrome del recomendado" es un fenómeno que no resulta lejano a los profesionales sanitarios. Se manifiesta en la aparición de complicaciones no habituales y de imprevistos en pacientes concretos en los que la intención es llevar a cabo una atención más esmerada y personalizada. Aparte de lo que pueda ser debido al azar, hay numerosos factores de la práctica clínica habitual que facilitan la manifestación de este síndrome. Pueden ser debidos a: la actitud del propio paciente, el empleo inadecuado de los recursos sanitarios, la falta de un buen registro de datos en la historia clínica y el cambio en la conducta habitual en el uso de los medios diagnósticos y en el tratamiento de estos enfermos.

Es preciso tomar conciencia de esta realidad para poder ir corrigiéndola, poco a poco. El medio más eficaz para prevenir el "síndrome del recomendado" es llevar a cabo una práctica clínica fundamentada en unos conocimientos clínicos sólidos. Y, a la vez, intentar mantener las líneas de conducta habituales, similares a las que se tiene con los demás enfermos.
9. Tannock IF. False-positive results in clinical trials: multiple significance tests and the problem of unreported comparisons. J Natl Cancer Inst 1996; 88: 206-7.

10. Lang TA, Sesic M. How to report statistics in medicine. Philadelphia: American College of Physicians; 1997.

11. Bartelink H. Is neoadjuvant chemotherapy the answer for bladder cancer. Lancet 1999; 354: 526-7.

12. Rochon PA, Gurwitz JH. Drug therapy. Lancet 1995; 346: 32-6.

13. Tannock IF. Treating the patient, not just the cancer. N Engl J Med 1987; 317: 1534-5.

14. Guyatt GH, Sackett DL, Cook DJ. How to use an article about therapy or prevention: Are the results of the study valid? JAMA 1993; 270: 2598-601.

15. Norton L. High dose chemotherapy for breast cancer: how do you know? J Clin Oncol 2001; 19: 2769-70.

16. Vallvé C. La buena práctica clínica y la caja de Pandora. La fiabilidad de los datos. Med Clin (Barc) 1997; 108: 65-7.

17. Harbour R, Miller J. A new system for grading recommendations in evidence based guidelines. BMJ 2001; 323: 334-6. 